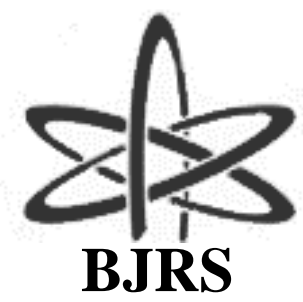

BRAZILIAN JOURNAL

$\mathrm{OF}$

RADIATION SCIENCES

03-1A (2015) 01-14

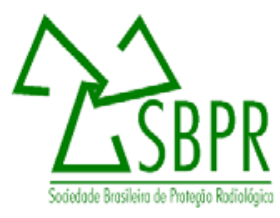

\title{
Levantamento bibliográfico sobre metodologias para elaboração de um banco de dados da saúde da população em casos de ocorrências de câncer
}

\author{
C. C. Cavinato ${ }^{\mathrm{a}}$; D. A. Andrade ${ }^{\mathrm{a}}$; M. D. P. E. Diz ${ }^{\mathrm{b}}$; G. Sabundjian ${ }^{\mathrm{a}}$ \\ ${ }^{a}$ Centro de Engenharia Nuclear, Instituto de Pesquisas Energéticas e Nucleares, 05508-000, São Paulo-SP, Brasil \\ ${ }^{b}$ Serviço de Oncologia Clínica, Instituto do Câncer do Estado de São Paulo Octavio Frias de Oliveira, 01246-000, \\ São Paulo-SP, Brasil \\ ccavinato@usp.br
}

\begin{abstract}
RESUMO
As fontes alternativas de energia, incluindo a nuclear, apresentam vantagens com relação às externalidades, as quais podem ser identificadas e relacionadas com o termo, custo ambiental. Este termo, por sua vez, é uma externalidade negativa, que de alguma forma prejudica o meio ambiente e é convertida em termos econômicos, para então poder ser comparada aos demais custos de uma ação e/ou empreendimento. A fim de efetuar os cálculos em questão, são utilizados alguns softwares específicos, os quais possibilitam a conversão dos danos em termos econômicos e a inclusão do custo ambiental na análise de custo de determinado projeto. Uma das dificuldades encontradas na utilização destes softwares tem sido com relação a alguns dados de entrada, como por exemplo, os relativos à saúde da população em torno da instalação nuclear, que são muito deficientes. O objetivo deste trabalho é fazer um levantamento teórico correspondente às metodologias existentes e utilizadas para a elaboração de banco de dados de saúde pública no Brasil. A partir das metodologias encontradas para a formação deste tipo de banco de dados, posteriormente será desenvolvida uma metodologia focando na saúde (câncer fatal e não fatal) da população circunvizinha a uma instalação nuclear, para fins de cálculo do custo ambiental da mesma. Essa será aplicada ao público interno do Instituto de Pesquisas Energéticas e Nucleares (IPEN), como um pré-teste, para aquisição das informações de saúde desejadas.
\end{abstract}

Palavras-chave: Base de dados, energia nuclear, saúde pública. 


\section{INTRODUÇÃO}

No Brasil, a utilização de usinas nucleares cresceu nas últimas décadas, mas ainda é pequena em comparação com outros países ao redor do mundo. Recentemente, o governo brasileiro manifestou interesse em aprovar o Plano Nacional de Energia Nuclear, que começou com a decisão da retomada da construção da usina nuclear Angra 3 e, a partir deste, fazer um escalonamento para que, a cada dois ou três anos, seja implantada uma nova usina de pequeno porte [1].

As fontes alternativas de energia, incluindo a nuclear, apresentam vantagens com relação às externalidades. Externalidade é um termo que representa os efeitos colaterais da produção de bens ou serviços sobre outras pessoas que não estão diretamente envolvidas com a atividade. As externalidades podem ter efeitos positivos ou negativos, podendo gerar custos ou benefícios para a sociedade. Elas podem ser identificadas e relacionadas com o termo custo ambiental. O custo ambiental é uma externalidade negativa que, de alguma forma, prejudica o meio ambiente e é convertida em termos econômicos, para então poder ser comparada aos demais custos de uma ação e/ou empreendimento [2].

A fim de efetuar os cálculos em questão são utilizados alguns softwares específicos, os quais possibilitam a conversão dos danos em termos econômicos e a inclusão do custo ambiental na análise de custo de determinado projeto [3]. Uma das dificuldades encontradas na utilização destes softwares tem sido com relação a alguns dados de entrada, como por exemplo, os relativos à saúde da população em torno da instalação nuclear, que são muito deficientes.

O objetivo deste trabalho é fazer um levantamento teórico correspondente às metodologias existentes e utilizadas para a elaboração de banco de dados de saúde pública no Brasil. A partir das metodologias encontradas para a formação deste tipo de banco de dados, posteriormente será desenvolvida uma metodologia focando na saúde (câncer fatal e não fatal) da população circunvizinha a uma instalação nuclear, para fins de cálculo do custo ambiental da mesma. Essa será aplicada ao público interno do Instituto de Pesquisas Energéticas e Nucleares (IPEN), como um pré-teste, para aquisição das informações de saúde desejadas. 


\section{METODOLOGIAS EMPREGADAS PARA UTILIZAÇÃO DE DADOS DE SAÚDE PÚBLICA}

Para medir a ocorrência de agravos à saúde ou seus desfechos (óbito, por exemplo) são utilizadas fontes de informação como prontuários médicos, de ambulatórios e de hospitais e atestados de óbito. Os dados também podem ser coletados por meio de questionários ou entrevistas aplicados ao paciente ou a alguém relacionado a ele.

As características do evento de interesse, como incidência de determinada doença na população, por exemplo, estão diretamente relacionadas à escolha da fonte de informação. Inquéritos domiciliares com aplicação de questionários são utilizados, geralmente, quando se deseja focalizar a fase pré-clínica das doenças. Os registros hospitalares constituem a principal fonte de informação quando se quer obter informações relativas a doenças de tratamento hospitalar obrigatório. Quando o objetivo é a obtenção de dados relativos à mortalidade e às morbidades notificáveis, os sistemas rotineiros de registros, como o Sistema de Informação de Mortalidade (SIM) e o Sistema de Notificação de Doenças de Notificação Compulsória (SINAM), utilizados pela vigilância epidemiológica, são as melhores fontes de informação. Vale ressaltar que o SIM apresenta menor sub-registro em relação ao SINAM.

Dados obtidos diretamente pelo pesquisador (primários) para propósito específico de uma pesquisa são mais confiáveis que dados secundários, embora demande tempo e gastos para sua aquisição. Deve-se considerar, além disso, que as estatísticas disponíveis até o momento referem-se ao atendimento em serviços públicos de saúde, por exemplo.

A estatística é ferramenta básica para a definição de amostras e técnicas de análise, uma vez que a epidemiologia também lida com dados populacionais.

Variáveis a serem investigadas devem ser estabelecidas para o estudo de um agravo numa população ou grupo específico. Nas variáveis independentes, podem estar contidos fatores individuais (exposição ocupacional, hábitos alimentares, prática de atividade física, aspectos genéticos, entre outros) ou locais (indicadores socioeconômicos, saneamento, cobertura dos 
serviços de saúde, entre outros). Almeja-se compreender a relação destas variáveis com a variável dependente (doença em questão, por exemplo) [4].

Existem diferentes critérios para classificar os estudos epidemiológicos. Eles podem ser descritivos (quando levantam hipóteses) ou analíticos (quando testam hipóteses), por meio de comparações entre grupos. Os estudos também podem ser experimentais ou observacionais, no que se refere à intervenção por parte do pesquisador. Também podem ser transversais (representam um corte no tempo) ou longitudinais (implicam no acompanhamento do(s) grupo(s) estudado(s) em longo prazo), quando o critério aplicado é o tempo.

A probabilidade (ou risco) de ocorrer um determinado evento entre indivíduos expostos a um fator é expressa pela incidência de um agravo numa população. Indica o risco absoluto, não permitindo estimar se a exposição está ou não associada à ocorrência do agravo investigado. Apenas o risco relativo permite estimar essa associação, o qual expressa uma comparação entre o risco de adoecer entre grupos expostos e não expostos a um determinado fator.

Em seu aspecto mais amplo, o estudo epidemiológico gira em torno da busca da causalidade. O conceito de causa é trabalhado no contexto de rede multicausal, dada sua complexidade [4].

\subsection{SISTEMAS DE INFORMAÇÃO EM SAÚDE}

Os grandes sistemas de informação em saúde, de análises e estimativas, bem como de pesquisas e estudos epidemiológicos, fornecem informações para a tomada de decisões em sistemas de vigilância.

O sistema de morbidade por câncer, com informações oportunas e de qualidade, é consolidado com base nas informações de 23 Registros de Câncer de Base Populacional (RCBP), alimentados por uma rede de 282 Registros Hospitalares de Câncer (RHC). Agrega-se a esse sistema, o SIM, para a elaboração da estimativa de 19 tipos de câncer apresentada para o território nacional, estados e capitais, por gênero [5].

\subsubsection{REGISTRO HOSPITALAR DE CÂNCER}


O Registro Hospitalar de Câncer (RHC) é uma fonte de informações sistemáticas dos casos novos de neoplasias malignas, tratados por determinado hospital. Um dos seus objetivos principais é cadastrar todos os casos novos atendidos e, então, conhecer a assistência prestada aos pacientes. Assim, pelo conhecimento da assistência oferecida, procura-se atingir a melhoria da atenção médica dispensada ao paciente oncológico.

Outras finalidades também importantes de um RHC são: servir como fonte de informações para o planejamento administrativo da Instituição; efetuar um controle eficiente do seguimento dos pacientes atendidos (possibilitando a análise de sobrevida destes); permitir a troca de informações com outros registros hospitalares e a organização de estudos colaborativos; estimular investigações clínicas sobre o câncer; e, estimular a melhoria do prontuário médico, principal fonte de dados para o RHC.

Conforme a Classificação Internacional de Doenças para Oncologia (CID-O) [6], o diagnóstico morfológico da neoplasia é o principal critério para a escolha dos casos a serem cadastrados no RHC, ressaltando tratar-se sempre de caso novo.

No Estado de São Paulo, por exemplo, existem Instituições que trabalharam na criação de Boletins RHC. Em 2000, a Instituição que recebeu a responsabilidade de coordenar a implantação do RHC para o Estado de São Paulo foi a Fundação Oncocentro de São Paulo (FOSP) e, desde então, executa tal tarefa.

Uma vez que o Serviço de Arquivo Médico (SAME) do hospital será a principal fonte de dados para o RHC, a integração com tal serviço é fundamental [7].

Para a compilação de dados do RHC faz-se necessário utilizar software específico [7]. Um exemplo de software utilizado para esta finalidade é o IntegradorRHC, aplicativo desenvolvido pelo Instituto Nacional do Câncer José Alencar Gomes da Silva (INCA). O IntegradorRHC cria um banco de dados nacional da incidência de câncer no Brasil, possibilitando, entre outras ações importantes, a orientação de políticas públicas que atuem na melhoria dos quadros apresentados. O cadastro neste aplicativo só é permitido aos coordenadores dos RHC, coordenadores estaduais e coordenadores nacionais $[8,9]$.

Outra ferramenta utilizada para trabalhar com dados de saúde é o TabNet, um aplicativo desenvolvido pelo Departamento de Informática do Sistema Único de Saúde (DATASUS), disponibilizado pela Secretaria Municipal da Saúde de São Paulo. Esse aplicativo possibilita o 
acesso às bases de dados de população e dos sistemas de informações (câncer, por exemplo) do Sistema Único de Saúde (SUS). Além disso, permite a realização de tabulações, cruzando-se diversas variáveis (bases de dados são atualizadas periodicamente), segundo o interesse do usuário [10].

\subsubsection{REGISTRO DE CÂNCER DE BASE POPULACIONAL}

Desde a sua implantação oficial, em janeiro de 1969, o Registro de Câncer de Base Populacional de São Paulo (RCBP-SP) funciona na Faculdade de Saúde Pública (FSP) da Universidade de São Paulo (USP). Além disso, o RCBP-SP recebe apoio técnico e financeiro da Secretaria Municipal de Saúde de São Paulo, por meio de convênio específico.

Os RCBP consistem em sistemas de informações específicos para a Oncologia, os quais coletam, classificam e analisam os casos novos de câncer diagnosticados em moradores de uma área geográfica bem definida. Desta forma, permite-se conhecer a incidência de câncer daquela área. Para a definição dos fatores etiológicos relacionados ao câncer, seus dados de incidência são fundamentais. Estes também contribuem para a definição de prioridades nas ações de prevenção, diagnóstico precoce e tratamento adequado aos pacientes com câncer [11].

Dependendo da ocorrência de novos tumores malignos, uma mesma pessoa poderá ser registrada várias vezes, em topografias distintas.

Os dados do Município de São Paulo são coletados ativamente em mais de 300 fontes, utilizando ficha padronizada. Algumas destas fontes de informações são hospitais gerais e especializados, clínicas, serviços de quimioterapia e/ou radioterapia, laboratórios de anatomia patológica, casas de repouso, serviços de autópsia, entre outras. Complementam-se as informações com dados referentes às Declarações de Óbitos (DO) fornecidas pelo Programa de Aprimoramento das Informações de Mortalidade no Município de São Paulo (PRO-AIM) e Fundação Sistema Estadual de Análise de Dados (SEADE) [12].

Eventuais comparações temporais devem ser analisadas de forma mais criteriosa, recomendandose consulta ao site do RCBP-SP, em virtude da possibilidade de variação dos casos novos de câncer coletados junto às diferentes fontes [13].

\subsubsection{SISTEMA DE INFORMAÇÕES SOBRE MORTALIDADE}

International Joint Conference RADIO 2014

Gramado, RS, Brazil, August 26-29, 2014

SOCIEDADE BRASILEIRA DE PROTEÇÃO RADIOLÓGICA - SBPR 
Em 1975, foi implantado oficialmente no Brasil, o SIM, sendo, portanto, um sistema novo, comparado aos similares existentes em outros países, como os europeus, Estados Unidos, Canadá e algumas nações da América Latina [14].

Alguns dos usos do SIM são: destacar a principal causa de morte da área; identificar a região de uma cidade ou estado de maior mortalidade e as causas mais importantes; possibilitar a comparação de diferentes regiões e detectar as mais saudáveis; verificar a existência de grupos populacionais específicos de risco (por idade, sexo, áreas, etc.), mais afetados por determinadas doenças; constatar a existência de variações sazonais na mortalidade por algumas doenças, entre outros.

Desde 1976, a atuação do Centro Brasileiro de Classificação de Doenças tem sido de grande relevância para a consecução dos objetivos do SIM, no que se refere à padronização de conceitos, preparação e divulgação de material instrucional. Além disso, tem importância na formação de recursos humanos, principalmente quanto ao uso da Classificação Internacional de Doenças (CID).

O aprimoramento do SIM também vem sendo estimulado, desde 1996, por meio da Rede Interagencial de Informações para a Saúde (RIPSA), para que o Brasil possa ter, cada vez mais, dados melhores de mortalidade e, consequentemente, indicadores de saúde mais fidedignos. As informações produzidas devem ser completas e de boa qualidade.

Acredita-se que, no futuro, o SIM terá superado todos os obstáculos, dos pontos de vista quantitativo e qualitativo. Assim, passará a ser, de fato, um instrumento valioso como produtor de insumos insubstituíveis para a tomada de decisões governamentais adequadas, visando à melhoria da saúde da população [14].

\section{DADOS DE CÂNCER}

Hoje, o câncer é reconhecido como um problema de saúde pública, o qual requer uma abordagem multidisciplinar. Vale relembrar que câncer é o nome dado a um conjunto de mais de 100 tipos diferentes de doenças que têm em comum, o crescimento desordenado de células 
anormais com potencial invasivo. Também é importante enfatizar que sua origem se dá por condições multifatoriais, podendo tais fatores agir em conjunto ou em sequência para iniciar ou promover o câncer [5].

Alguns tipos de câncer podem ser evitados pela eliminação da exposição aos fatores determinantes, considerando que o desenvolvimento da maioria dos cânceres requer múltiplas etapas que ocorrem ao longo de muitos anos. Tem-se uma condição mais favorável para seu tratamento, objetivando a cura, desde que o potencial de malignidade seja detectado na fase inicial da doença.

Em 2012, houve 14,1 milhões de casos novos de câncer e um total de 8,2 milhões de mortes por câncer, em todo o mundo. Estes dados são estimativas mundiais do projeto Globocan 2012, da Agência Internacional para Pesquisa em Câncer (IARC, do inglês International Agency for Research on Cancer), da Organização Mundial da Saúde (OMS). Se medidas preventivas não forem amplamente aplicadas, o número de casos de câncer crescerá ainda mais em países desenvolvidos e continuará aumentando nos países em desenvolvimento.

Os tipos de câncer mais frequentes na população masculina de países desenvolvidos foram próstata, pulmão e cólon e reto; entre as mulheres foram mama, cólon e reto e pulmão. Nos países em desenvolvimento, cânceres de pulmão, estômago e fígado foram os três mais frequentes em homens; e, mama, colo do útero e pulmão, nas mulheres [5].

No entanto, podem ser prevenidos mais da metade dos casos previstos, ampliando-se as ações de prevenção, como combate à obesidade, ao alcoolismo e ao tabagismo. Também associados ao câncer estão fatores como radiações solar e ionizante (esta última, provinda de aceleradores lineares clínicos utilizados em radioterapia, por exemplo), poluição do ar e recusa de amamentação por parte das mães [9,15].

No Brasil, a estimativa de ocorrência de câncer aponta para aproximadamente 576 mil casos novos de câncer, para o ano de 2014 (válida também para o ano de 2015). Nestes dados, estão incluídos os casos de pele não melanoma, reforçando a magnitude do problema do câncer no país. O câncer mais incidente na população brasileira será o de pele do tipo não melanoma (182 mil casos novos), seguido pelos tumores de próstata (69 mil), mama feminina (57 mil), cólon e reto (33 mil), pulmão (27 mil), estômago $(20$ mil) e colo do útero $(15$ mil $)$. Em 2030, em razão do crescimento e do envelhecimento da população, bem como da redução na 
mortalidade infantil e nas mortes por doenças infecciosas em países em desenvolvimento, a carga global será de 21,4 milhões de casos novos de câncer e 13,2 milhões de mortes por câncer [5].

No Município de São Paulo, os últimos dados disponíveis sobre casos notificados de câncer são de 2011 e totalizam 43.444 casos, segundo o RCBP-SP [16].

\section{VIGILÂNCIA DO CÂNCER RELACIONADA AO TRABALHO}

Agentes cancerígenos como o amianto, a sílica, solventes aromáticos (benzeno, por exemplo), metais pesados (níquel e cromo, por exemplo), a radiação ionizante e alguns agrotóxicos, podem ser encontrados nos ambientes de trabalho. O efeito desses agentes pode ser potencializado se houver exposição a outros fatores de risco para câncer, como a poluição ambiental, dieta rica em gorduras trans, consumo exagerado de álcool e o tabagismo. O câncer de pulmão, os mesoteliomas, o câncer de pele, o de bexiga e as leucemias são os tipos mais frequentes de câncer relacionado ao trabalho.

A interação da radiação ionizante com os seres vivos pode levar a alterações teratogênicas e até à morte e, portanto, tais efeitos não podem ser considerados inócuos. Pelo fato da radiação ionizante apresentar riscos à saúde, deve ser usada de forma consciente, de acordo com os seus benefícios. Alguns destes benefícios são os seguintes: geração de energia elétrica (por meio das usinas nucleares), radioesterilização de materiais médico-hospitalares, irradiação de alimentos, tratamento de doenças por meio da radioterapia e da braquiterapia, diagnóstico médico (radiografia, tomografia, mamografia e mapeamento com radiofármacos), dentre outros usos pacíficos.

Estão ocupacionalmente expostos à radiação ionizante, indivíduos que trabalham na indústria nuclear ou em torno de equipamentos que emitem radiação (em instituições médicas ou laboratórios, por exemplo). A média anual de dose efetiva de raios $\mathrm{X}$ e gama provenientes de fontes naturais é de 0,5 a 5,0 mSv. Em países desenvolvidos, os procedimentos médicos resultam em uma dose efetiva anual de 1 a $2 \mathrm{mSv}$ (2/3 destes procedimentos são decorrentes de 
diagnósticos utilizando radiografias). A dose efetiva anual para trabalhadores monitorados está entre 1 e $10 \mathrm{mSv}$ [17].

A Comissão Nacional de Energia Nuclear (CNEN), na Resolução de 17 de dezembro de 2004, publicada em Diário Oficial da União (D.O. 06/01/2005), descreve as "Diretrizes Básicas de Radioproteção” [18]. Pode-se encontrar nesta Resolução, a limitação de dose individual (dose efetiva e dose equivalente) e monitoramento da avaliação da exposição ocupacional [17].

Considerando a possibilidade da correlação da exposição ocupacional e não ocupacional (indivíduos do público) à radiação ionizante, com a incidência de alguns tipos de câncer e, com base nas metodologias empregadas para aquisição de dados de saúde pública encontradas na literatura, será desenvolvida uma metodologia a ser aplicada ao público interno do Instituto de Pesquisas Energéticas e Nucleares (IPEN). Inicialmente, esta metodologia será a aplicação de um pré-teste para aquisição de informações sobre a saúde dos funcionários do Instituto.

Este trabalho está sendo realizado em colaboração com o Instituto do Câncer do Estado de São Paulo Octavio Frias de Oliveira (ICESP). A metodologia utilizada no Serviço de Oncologia Clínica deste Instituto, para computar dados de câncer no RHC implantado no ICESP, servirá de base para a elaboração da metodologia a ser desenvolvida nesta pesquisa. Além disso, por meio do trabalho que será desenvolvido no IPEN, serão disponibilizadas informações sobre casos de câncer fatal e não fatal em instalações radiativas e nucleares, bem como em seus arredores, as quais serão agregadas ao banco de dados do ICESP.

\section{CONCLUSÕES}

Diferentes formas de obtenção de dados são utilizadas em saúde pública, as quais são de extrema importância para o levantamento de informações que auxiliarão na análise da qualidade de vida da população.

Procura-se, cada vez mais, aprimorar os sistemas de informação em saúde disponíveis e conscientizar os responsáveis pelo fornecimento de tais informações, sobre a importância de se manter bancos de dados de saúde com números fidedignos de casos de doenças, como o câncer. 
Com isso, pretende-se adotar medidas preventivas e corretivas efetivas para a redução da morbimortalidade da doença em questão.

Com base nas metodologias levantadas na literatura e com a experiência adquirida em parceria com o ICESP, será possível desenvolver um banco de dados sobre casos de câncer fatal e não fatal, ocorridos em trabalhadores de instalações radiativas e nucleares, bem como na população circunvizinha. Com este banco de dados, que será elaborado futuramente, pretende-se mostrar que o sistema de segurança destas instalações, no que se refere à radiação ionizante, é bastante eficaz. A expectativa com a realização deste trabalho é que o número de casos de câncer oriundos da exposição à radiação ionizante seja equiparado aos casos decorrentes de outros fatores de risco, como o tabagismo, por exemplo. Em contrapartida, se houver alguma discrepância, sendo maior o número de casos relacionados com a exposição à radiação ionizante, esta pesquisa contribuirá para a tomada de medidas corretivas de proteção radiológica, nos locais onde tal ação se fizer necessária.

\section{AGRADECIMENTOS}

À ELETROBRAS ELETRONUCLEAR/Coordenação de Aperfeiçoamento de Pessoal de Nível Superior (Capes) e ao Centro de Engenharia Nuclear do Instituto de Pesquisas Energéticas e Nucleares, pelo apoio financeiro. Ao Instituto do Câncer do Estado de São Paulo Octavio Frias de Oliveira, pela colaboração no desenvolvimento do trabalho. 


\section{REFERÊNCIAS}

1. CAminOtO, J. Brasil quer mais sete usinas atômicas. O Estado de São Paulo. São Paulo, Brasil. 8 mar. 2006. Disponível em:

$<$ http://www2.senado.leg.br/bdsf/bitstream/handle/id/317781/noticia.htm?sequence=1 > .

Último acesso: 16 fev. 2015.

2. THOMAS, J.M.; CALLAN, S.J. Economia ambiental: aplicações, políticas e teoria. São Paulo: Cengage Learning, 2010.

3. INTERNATIONAL ATOMIC ENERGY AGENCY (IAEA). Department of nuclear energy: SIMPACTS. Vienna, Austria. 2010. Disponível em:

$<$ http://www.iaea.org/OurWork/ST/NE/Pess/PESSenergymodels.shtml>. Último acesso: 16 fev. 2015.

4. ROCHA, A.A.; CESAR, C.L.G. Saúde pública. São Paulo: Atheneu, 2008.

5. INSTITUTO NACIONAL DE CÂNCER JOSÉ ALENCAR GOMES DA SILVA (INCA). Estimativa, Incidência de câncer no Brasil, Introdução. Rio de Janeiro, Brasil. 2014. Disponível em: <http://www.inca.gov.br/estimativa/2014/index.asp?ID=2>. Último acesso: 16 fev. 2015.

6. ORGANIZAÇÃO MUNDIAL DA SAÚDE (OMS). CID-O: Classificação Internacional de Doenças para Oncologia. Editora da Universidade de São Paulo/Fundação Oncocentro de São Paulo: São Paulo, 2005. 
7. SECRETARIA DO ESTADO DA SAÚDE DE SÃO PAULO/ FUNDAÇÃO ONCOCENTRO DE SÃO PAULO. Sobrevida de pacientes com câncer no Estado de São Paulo: seis anos de seguimento pelo Registro Hospitalar de Câncer, 2000 a 2005. Cadernos FOSP, v. 5, p. 1-166, 2009.

8. INSTITUTO NACIONAL DE CÂNCER JOSÉ ALENCAR GOMES DA SILVA (INCA). Estatísticas do câncer, Vigilância do câncer e de fatores de risco, Sistemas de informação. Rio de Janeiro, Brasil. 2014. Disponível em: <http://www1.inca.gov.br/vigilancia/sistemas.asp>. Último acesso: 16 fev. 2015.

9. D’ÂNGELO, C. A nova abordagem do câncer. Rede Câncer, v. 1, p.10-11, 2007.

10. PREFEITURA DE SÃO PAULO. Secretaria Municipal da Saúde, TabNet. São Paulo, Brasil. $2014 . \quad$ Disponível em: $<$ http://www.prefeitura.sp.gov.br/cidade/secretarias/saude/tabnet>. Último acesso: 16 fev. 2015.

11. PREFEITURA DE SÃO PAULO. Secretaria Municipal da Saúde, Registro de Câncer de Base Populacional de São Paulo. São Paulo, Brasil. 2014. Disponível em: $<$ http://www.prefeitura.sp.gov.br/cidade/secretarias/saude/epidemiologia_e_informacao/inde x.php?p=30177> . Último acesso: 16 fev. 2015.

12. PREFEITURA DE SÃO PAULO. Secretaria Municipal da Saúde, Notas técnicas, Registro de câncer. São Paulo, Brasil. 2014. Disponível em: $<$ http://www.prefeitura.sp.gov.br/cidade/secretarias/saude/tabnet/index.php?p=14018> . Último acesso: 16 fev. 2015. 
13. REGISTRO DE CÂNCER DE BASE POPULACIONAL DE SÃO PAULO (RCBP-SP). Publicações. São Paulo, Brasil. 2014. Disponível em: <http://www.fsp.usp.br/rcsp/publicacoes>. Último acesso: 16 fev. 2015.

14. LAURENTI, R; MELLO JORGE, M.H.P; GOTLIEB, S.L.D. O Sistema de Informações sobre Mortalidade: passado, presente e futuro. São Paulo: CBCD/MS/USP/OPAS/OMS, 2006.

15. ESCOLA NACIONAL DE SAÚDE PÚBLICA SERGIO AROUCA (ENSP). Casos de câncer aumentam no mundo. RADIS, v. 138, p. 6, 2014.

16. PREFEITURA DE SÃO PAULO. Secretaria Municipal da Saúde, TabNet, Câncer, Registro de Câncer de Base Populacional (São Paulo). São Paulo, Brasil. 2011. Disponível em: $<$ http://tabnet.saude.prefeitura.sp.gov.br/cgi/tabcgi.exe?secretarias/saude/TABNET/CA/canc er.def>. Último acesso: 16 fev. 2015.

17. INSTITUTO NACIONAL DE CÂNCER JOSÉ ALENCAR GOMES DA SILVA (INCA). Coordenação de prevenção e vigilância, Vigilância do câncer relacionado ao trabalho e ao ambiente. INCA: Rio de Janeiro, 2010.

18. COMISSÃO NACIONAL DE ENERGIA NUCLEAR (CNEN). CNEN NN 3.01 Diretrizes básicas de proteção radiológica. Rio de Janeiro, Brasil. 2014. Disponível em: <http://www.cnen.gov.br/seguranca/normas/pdf/Nrm301.pdf>. Último acesso: 16 fev. 2015. 